Pacific Journal of Mathematics

LATTICE PATHS AND MULTIPLE BASIC HYPERGEOMETRIC 


\section{LATTICE PATHS AND MULTIPLE BASIC HYPERGEOMETRIC SERIES}

\section{A. K. Agarwal and David M. Bressoud}

Certain basic hypergeometric series with multiple indices of summation are interpreted as generating functions for weighted lattice paths. The approach uses ideas of William Burge and gives rise to identities analogous to the Rogers-Ramanujan identities.

Many of the generating function identities that have arisen in partition theory have natural extensions to multiple series. The classical example of this consists of the Rogers-Ramanujan identities:

$$
\sum_{m \geq 0} \frac{q^{m^{2}}}{(q)_{m}}=\prod\left(1-q^{n}\right)^{-1}, \quad n \geq 1, n \equiv \pm 1 \quad(\bmod 5),
$$

$$
\sum_{m \geq 0} \frac{q^{m^{2}+m}}{(q)_{m}}=\prod\left(1-q^{n}\right)^{-1}, \quad n \geq 2, n \equiv \pm 1 \quad(\bmod 5),
$$

where $(a)_{m}(a ; q)_{m}=(1-a)(1-a q) \cdots\left(1-a q^{m-1}\right)$.

A generalization of these identities to arbitrary odd modulus was found by George Andrews [And 1]: $(1 \leq i \leq k+1,1 \leq k)$

$$
\begin{aligned}
& \sum \frac{q^{m_{1}^{2}+\cdots+m_{k}^{2}+m_{i}+\cdots+m_{k}}}{(q)_{m_{1}-m_{2}} \cdots(q)_{m_{k-1}-m_{k}}(q)_{m_{k}}}=\prod\left(1-q^{n}\right)^{-1}, \\
& \quad m_{1} \geq m_{2} \geq \cdots \geq m_{k} \geq 0 ; n \geq 1, n \neq 0, \pm i(\bmod 2 k+3) .
\end{aligned}
$$

While each side of equations (0.1) and (0.2) is easily interpreted as a generating function for certain restricted partitions, the interpretation of the left-hand side of equation (0.3) presents more of a problem. It was William Burge [Bur 1, Bur 2] who demonstrated that the most natural interpretation is in terms of weighted binary words. He was then able to establish one-to-one correspondences between his binary words of weight $n$ and several different sets of partitions of $n$, thus providing new proofs of partition identities of Basil Gordon [Gor] and George Andrews [And 2].

Burge also gave interpretations of two other multiple basic hypergeometric series, and Andrews and Bressoud [A\&B] have interpreted a 
fourth multiple series and restated all of the conditions on the binary words as restrictions on weighted lattice paths. The switch to lattice paths was made because the restrictions become easier to describe and visualize.

In $\S 1$, we shall define our terminology and give Burge's interpretations of the three multiple basic hypergeometric series he considered. We shall also interpret some recently discovered series found by Agarwal, Andrews and Bressoud [AA\&B]. The proof that these new series are the generating functions for the appropriate classes of weighted lattice paths is given in $\S \S 2$ through 5 . In the sixth section, we establish a bijection between the appropriate class of lattice paths of weight $n$ and a set of partitions of $n$. This bijection provides a new proof of a partition identity of Agarwal and Andrews [A\&A].

1. The lattice paths. We shall be considering lattice paths of finite length lying in the first quadrant. All our paths will begin on the $y$-axis and terminate on the $x$-axis. Only three moves are allowed at each step:

northeast : from $(i, j)$ to $(i+1, j+1)$,

southeast: $\quad$ from $(i, j)$ to $(i+1, j-1)$, only allowed if $j>0$,

horizontal : from $(i, 0)$ to $(i+1,0)$, only allowed along $x$-axis.

All our lattice paths are either empty or terminate with a southeast step: from $(i, 1)$ to $(i+1,0)$.

In describing lattice paths, we shall use the following terminology:

PEAK: Either a vertex on the $y$-axis which is followed by a southeast step or a vertex preceded by a northeast step and followed by a southeast step.

VALLEY: A vertex preceded by a southeast step and followed by a northeast step. Note that a southeast step followed by a horizontal step followed by a northeast step does not constitute a valley.

MOUNTAIN: A section of the path which starts on either the $x$-or $y$-axis, which ends on the $x$-axis, and which does not touch the $x$-axis anywhere in between the endpoints. Every mountain has at least one peak and may have more than one.

RANGE: A section of the path which starts either on the $y$-axis or at a vertex preceded by a horizontal step, which ends either at the end of the path or at a vertex followed by a horizontal step, and which does not contain any horizontal steps. Every range includes at least one mountain and may have more than one. 
PLAIN: A section of path consisting of only horizontal steps which starts either on the $y$-axis or at a vertex preceded by a southeast step and ends at a vertex followed by a northeast step.

EXAMPLE. The following path has five peaks, three valleys, three mountains, two ranges and one plain.

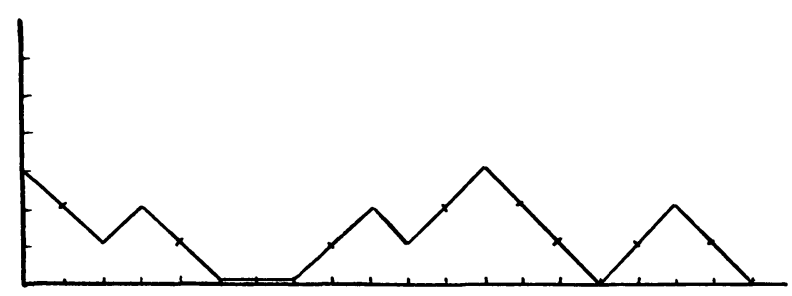

FIGURE 1

The HEIGHT of a vertex is its $y$-coordinate. The WEIGHT of a vertex is its $x$-coordinate. The WEIGHT OF A PATH is the sum of the weights of its peaks.

In the example given above, there are two peaks of height three and three of height two, two valleys of height one and one of height zero. The weight of this path is $0+3+9+12+17=41$.

We sum up what has been proved in [Bur 1, Bur 2].

Proposition 1.1 [Bur 1]. The multiple summation on the left side of equation (0.3) is the generating function for $A(k, i, n):=$ the number of lattice paths of weight $n$ which start at $(0, k+1-i)$, and have no peak above height $k$.

COROLlaRy 1.1. $A(k, i, n)=$ the number of partitions of $n$ into parts which are not congruent to 0 or $\pm i$ modulo $2 k+3$.

The next identity was first proved in [Bre 1]. For $1 \leq i \leq k$ :

$$
\begin{aligned}
\sum \frac{q^{m_{1}^{2}+\cdots+m_{k}^{2}+m_{1}+\cdots+m_{k}}}{(q)_{m_{1}-m_{2}} \cdots(q)_{m_{k-1}-m_{k}}\left(q^{2} ; q^{2}\right)_{m_{k}}}=\prod\left(1-q^{n}\right)^{-1}, \\
m_{1} \geq \cdots \geq m_{k} \geq 0 ; n \geq 1, n \neq 0, \pm i(\bmod 2 k+2) .
\end{aligned}
$$

Proposition 1.2 [Bur 1]. The multiple summation on the left side of equation (1.1) is the generating function for $B(k, i, n):=$ the number of lattice paths of weight $n$ which start at $(0, k+1-i)$, have no peak 
above height $k$ and are such that every peak of height $k$ has weight congruent to $i-1$ modulo 2 .

COROLlaRY 1.2. $B(k, i, n)=$ the number of partitions of $n$ into parts not congruent to 0 or $\pm i$ modulo $2 k+2$.

The next identity was first proved in [Bre 2], equation (3.8). For $i \leq i \leq k+1$,

$$
\begin{array}{r}
\sum \frac{q^{2\left(m_{1}^{2}+\cdots+m_{k}^{2}+m_{1}+\cdots+m_{k}\right)}\left(-q^{1-2 m_{1}} ; q^{2}\right)_{m_{1}}}{\left(q^{2} ; q^{2}\right)_{m_{1}-m_{2}} \cdot\left(q^{2} ; q^{2}\right)_{m_{k-1}-m_{k}}\left(q^{2} ; q^{2}\right)_{m_{k}}}=\prod\left(1-q^{n}\right)^{-1} \\
m_{1} \geq \cdots \geq m_{k} \geq 0 ; n \geq 1, n \neq \equiv 2(\bmod 4) \\
\not \neq 0, \pm(2 i-1)(\bmod 4 k+4)
\end{array}
$$

Proposition 1.3 [Bur 2]. The multiple summation on the left side of equation (1.2) is the generating function for $C(k, i, n):=$ the number of lattice paths of weight $n$ which start at $(0,2 k+2-2 i)$, have maximum height $2 k$ and are such that all plains have even length and all valleys have even weight.

COROLlaRY 1.3. $C(k, i, n)=$ the number of partitions of $n$ into parts not congruent to 2 modulo 4 nor congruent to 0 or $\pm(2 i-1)$ modulo $4 k+4$.

Our final set of identities was proved in [AA\&B]. If $k \geq 3$ is odd, $1 \leq i \leq(k+1) / 2$, and $r=(k-1) / 2$, then

$$
\begin{gathered}
\sum \frac{q^{m_{1}^{2}+\cdots+m_{r}^{2}+m_{1}+\cdots+m_{i-1}+2 m_{1}+\cdots+2 m_{r}}}{(q)_{m_{1}-m_{2}} \cdots(q)_{m_{r-1}-m_{r}}(q)_{m_{r}}\left(q ; q^{2}\right)_{m_{r}+1}}=\prod\left(1-q^{n}\right)^{-1}, \\
m_{1} \geq \cdots \geq m_{r} \geq 0 ; n \geq 1, n \neq 0, \pm 2 i(\bmod 4 k+2) .
\end{gathered}
$$

If $k \geq 2$ is even, $1 \leq i \leq(k+2) / 2$, and $r=k / 2$, then

$$
\begin{aligned}
& \sum \frac{q^{m_{1}^{2}+\cdots+m_{r}^{2}+m_{1}+\cdots+m_{i-1}+2 m_{1}+\cdots+2 m_{r}+m_{r}\left(m_{r}-1\right) / 2}}{(q)_{m_{1}-m_{2}} \cdots(q)_{m_{r-1}-m_{r}}(q)_{m_{r}}\left(q ; q^{2}\right)_{m_{r}+1}} \\
& =\prod\left(1-q^{n}\right)^{-1}, \quad m_{1} \geq \cdots \geq m_{r} \geq 0 ; \\
& n \geq 1, n \neq 0, \pm 2 i(\bmod 4 k+2) .
\end{aligned}
$$

If $k \geq 3$ is odd, $(k+1) / 2 \leq i \leq k-1$, and $r=(k-1) / 2$, then

$$
\begin{aligned}
& \sum \frac{q^{m_{1}^{2}+\cdots+m_{r}^{2}-m_{1} \cdots-m_{k-1}}\left(1-q^{m_{k-i}}\right)}{(q)_{m_{1}-m_{2}} \cdots(q)_{m_{r-1}-m_{r}}(q)_{m_{r}}\left(q ; q^{2}\right)_{m_{r}}}=\prod\left(1-q^{n}\right)^{-1}, \\
& m_{1} \geq \cdots \geq m_{r} \geq 0 ; n \geq 1, n \neq 0, \pm 2 i(\bmod 4 k+2) .
\end{aligned}
$$


If $k \geq 2$ is even, $k / 2 \leq i \leq k-1$, and $r=k / 2$, then

$$
\begin{aligned}
& \sum \frac{q^{m_{1}^{2}+\cdots+m_{r}^{2}-m_{1}-\cdots-m_{k-1}+m_{r}\left(m_{r}-1\right) / 2}\left(1-q^{m_{k-1}}\right)}{(q)_{m_{1}-m_{2}} \cdots(q)_{m_{r-1}-m_{r}}(q)_{m_{r}}\left(q ; q^{2}\right)_{m_{r}}} \\
& =\prod\left(1-q^{n}\right)^{-1}, \quad m_{1} \geq \cdots \geq m_{r} \geq 0 ; \\
& n \geq 1, n \neq 0, \pm 2 i(\bmod 4 k+2) .
\end{aligned}
$$

If $k \geq 3$ is odd, $i=k$, and $r=(k-1) / 2$, then

$$
\begin{aligned}
& \sum \frac{q^{m_{1}^{2}+\cdots+m_{r}^{2}}}{(q)_{m_{1}-m_{2}} \cdots(q)_{m_{r-1} m_{r}}(q)_{m_{r}}\left(q ; q^{2}\right)_{m_{r}}}=\prod\left(1-q^{n}\right)^{-1} \\
& m_{1} \geq \cdots \geq m_{r} \geq 0 ; n \geq 1, n \neq \equiv 0, \pm 2 k(\bmod 4 k+2)
\end{aligned}
$$

If $k \geq 2$ is even, $i=k$, and $r=k / 2$, then

$$
\begin{aligned}
\sum \frac{q^{m_{1}^{2}+\cdots+m_{r}^{2}+m_{r}\left(m_{r}-1\right) / 2}}{(q)_{m_{1}-m_{2}} \cdots(q)_{m_{r-1}-m_{r}}(q)_{m_{r}}\left(q ; q^{2}\right)_{m_{r}}}=\prod\left(1-q^{n}\right)^{-1} \\
m_{1} \geq \cdots \geq m_{r} \geq 0 ; n \geq 1, n \not \equiv 0, \pm 2 k(\bmod 4 k+2) .
\end{aligned}
$$

The following proposition will be proved in the next sections.

Proposition 1.4. The multiple summations on the left-hand sides of equations (1.3)-(1.8) can each be interpreted, in the appropriate range, as the generating function for $D(k, i, n):=$ the number of lattice paths of weight $n$ which start at $(0, k-i)$ and have no valley above height $k-3$. When $k=2$ this means there are no valleys.

COROLlaRY 1.4. $D(k, i, n)=$ the number of partitions of $n$ into parts not congruent to 0 or $\pm 2 i$ modulo $4 k+2$.

2. Proof of Proposition 1.4 when $r=1$. We shall start with equation (1.7) in the special case where $k=3$ (and thus $i=3$ ):

$$
\begin{aligned}
& \sum \frac{q^{m^{2}}}{(q)_{m}\left(q ; q^{2}\right)_{m}}=\prod\left(1-q^{n}\right)^{-1} \\
& n \geq 1, n \neq 0, \pm 6(\bmod 14) .
\end{aligned}
$$

The factor $q^{m^{2}}$ generates the lattice path of $m$ peaks starting at $(0,0)$ and terminating at $(2 m, 0)$. If $m=4$, then the path begins as:

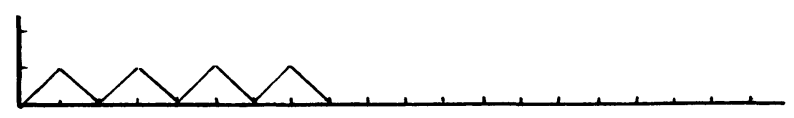


The factor $(q)_{m}^{-1}$ generates $m$ non-negative parts, say $a_{1} \geq a_{2} \geq$ $\cdots \geq a_{m} \geq 0$, which are encoded by inserting $a_{m}$ horizontal steps in front of the first mountain and $a_{i}-a_{i+1}$ horizontal steps in front of the $(m-i+1)$ st mountain, $1 \leq i \leq m$. If $a_{1}=3, a_{2}=1, a_{3}=1$, $a_{4}=0$, then our example becomes:

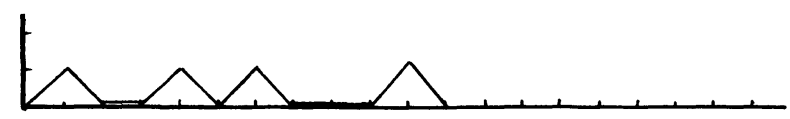

FIGURE 3

The factor $\left(q ; q^{2}\right)_{m}^{-1}$ generates non-negative multiples of $2 i-1,1 \leq$ $i \leq m$, say $b_{1} * 1, b_{2} * 3, \ldots, b_{m} *(2 m-1)$. This is encoded by having the $i$ th peak grow to height $b_{m-i+1}+1$. Each increase by one in the height of a given peak increases its weight by one and the weight of each subsequent peak by two. If $b_{1}=3, b_{2}=1, b_{3}=2, b_{4}=0$, then our example becomes:

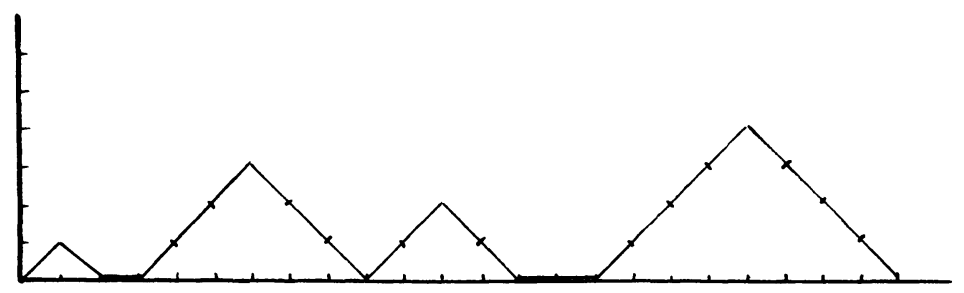

FIGURE 4

Every lattice path starting at $(0,0)$ with all valleys at height 0 is uniquely generated in this fashion.

Equation (1.8) with $k=2$ (and thus $i=2$ )

$$
\begin{aligned}
& \sum \frac{q^{m^{2}+m(m-1) / 2}}{(q)_{m}\left(q ; q^{2}\right)_{m}}=\prod\left(1-q^{n}\right)^{-1} \\
& n \geq 1, n \neq 0, \pm 4(\bmod 10),
\end{aligned}
$$

is treated in exactly the same manner except that the factor of $q^{m(m-1) / 2}$ inserts a horizontal step between each of the peaks so that there are no valleys. If we keep the same values of the $a$ 's and $b$ 's, the 
lattice path generated is

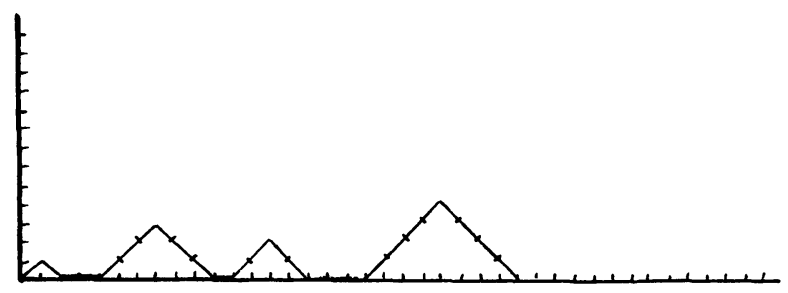

FIGURE 5

The case $k=i=2$ also arises from equation (1.4) where the generating function becomes

$$
\begin{aligned}
\sum \frac{q^{m^{2}+m+m(m-1) / 2}}{(q)_{m}\left(q ; q^{2}\right)_{m+1}}=\prod\left(1-q^{n}\right)^{-1} & \\
& n \geq 1, n \neq \equiv, \pm 4(\bmod 10) .
\end{aligned}
$$

The extra factor of $q^{m}$ puts a horizontal step in front of the first peak. The extra factor of $\left(1-q^{2 m+1}\right)^{-1}$ introduces a non-negative multiple of $2 m+1$, say $b_{m+1} *(2 m+1)$, which is encoded as a mountain of height $b_{m+1}$ inserted at the beginning of the lattice path.

The difference between the generating functions of (2.2) and (2.3) is that in (2.2) $m$ counts the number of ranges while in (2.3) $m$ counts the number of plains.

In the case $k=2, i=1$ of equation (1.4)

$$
\sum \frac{q^{m^{2}+2 m+m(m-1) / 2}}{(q)_{m}\left(q ; q^{2}\right) m+1}+\prod\left(1-q^{n}\right)^{-1}, \quad n \geq 1, n \neq \equiv 0, \pm 2 \quad(\bmod 10),
$$

we are bringing in an extra factor of $q^{m}$ which introduces a southeast step from $(0,1)$ to $(1,0)$ at the front of the lattice path.

We next consider equation (1.3) with $k=3$ and $i=1$ or 2 .

$$
\begin{aligned}
\sum \frac{q^{m^{2}+(3-i) m}}{(q)_{m}\left(q ; q^{2}\right)_{m+1}}=\prod\left(1-q^{n}\right)^{-1}, \\
n \geq 1, n \neq 0, \pm 2 i(\bmod 14) .
\end{aligned}
$$

This is interpreted essentially the same as the previous examples. We no longer have to have horizontal steps between peaks. If $i=2$ we insert a southeast step $(0,1)$ to $(1,0)$ at the beginning of the path. If $i=1$ then we insert two southeast steps $(0,2)$ to $(1,1)$ and $(1,1)$ to $(2,0)$ at beginning of the path. 
In equation (1.5) with $k=3$ and $i=2$, the generating function becomes

$$
\begin{array}{r}
\sum \frac{q^{m^{2}-m}\left(1-q^{m}\right)}{(q)_{m}\left(q ; q^{2}\right)_{m}}=\sum \frac{q^{m^{2}-m}}{(q)_{m}\left(q ; q^{2}\right)_{m}}-\sum \frac{q^{m^{2}}}{(q)_{m}\left(q ; q^{2}\right)_{m}} \\
=\prod\left(1-q^{n}\right)^{-1}, \quad n \geq 1, n \neq 0, \pm 4(\bmod 14) .
\end{array}
$$

As we have seen, the function

$$
\frac{q^{m^{2}}}{(q)_{m}\left(q ; q^{2}\right)_{m}}
$$

generates paths with $m$ peaks which start at $(0,0)$ and have no valleys above height 0 . To introduce the factor $q^{-m}$ we eliminate the first step and consider two cases: if the path began with a horizontal step, we now have a path with $m$ peaks which starts at $(0,0)$; if the path began with a northeast step, it still has $m$ peaks but now begins at $(0,1)$. When we subtract off the generating function for paths starting at $(0,0)$, we are left with the desired generating function.

Equation (1.6) with $k=2$ and $i=1$ is analyzed in exactly the same manner, except that now we have at least one horizontal step between each peak.

3. Proof of Proposition 1.4 for equations (1.7) and (1.8). The multiple summation in equation (1.7) is given by

$$
\sum \frac{q^{m_{1}^{2}+\cdots+m_{r}^{2}}}{(q)_{m_{1}-m_{2}} \cdots(q)_{m_{r-1}-m_{r}}(q)_{m_{r}}\left(q ; q^{2}\right)_{m_{r}}} .
$$

To build this up inductively, we shall need to specify what the $m_{j}$ 's are counting.

For each mountain in our path, we define as follows a multi-set of ordered pairs called the SET OF RELATIVE PEAKS of the mountain. We choose a peak of maximal height, the first element of the set is the ordered pair whose first coordinate is the height of the peak and whose second coordinate is 0 . As we proceed we ignore all peaks that have previously been chosen.

If there are any unchosen peaks left, we cut the mountain off at height one. This may have the effect of subdividing our mountain into several mountains. In fact, the number of mountains relative to height one will be one more than the number of valleys at height one. For each mountain relative to height one in which no peaks have been chosen, we choose a peak of maximal height and create a new element 
of the set of relative peaks whose first coordinate is the height of that peak and whose second coordinate is one.

Inductively, after creating all elements whose second coordinate is $H-1$, if any unchosen peaks remain we cut the mountains off at height $H$. The number of mountains relative to height $H$ will be one more than the number of valleys at or below height $H$ minus the number of peaks at or below height $H$. For each mountain relative to height $H$ in which no peaks have been chosen, we choose a peak of maximal height and create a new element of the set of relative peaks whose first coordinate is the height of that peak and whose second coordinate is $H$. Thus the mountain given below has $\{(5,0),(5,1),(2,1),(3,2)\}$ as its set of relative peaks.

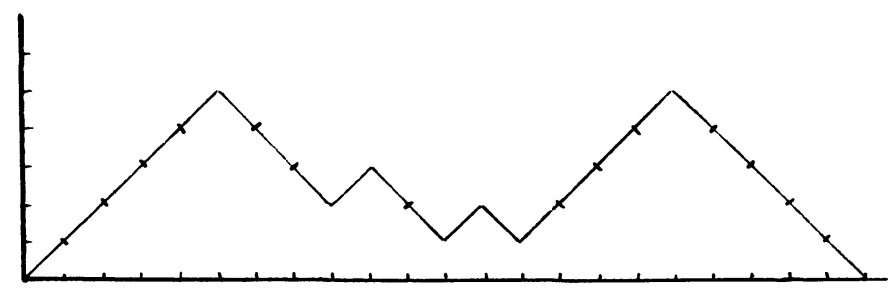

Figure 6

Note that while the order in which peaks are chosen is not unique, the set generated is unique.

The set of relative peaks of a lattice path is the multi-set union of the sets of relative peaks of all mountains in the lattice path. For the path given below, the set of relative peaks is $\{(3,0),(3,0),(2,0),(2,1)$, $(2,1)\}$.

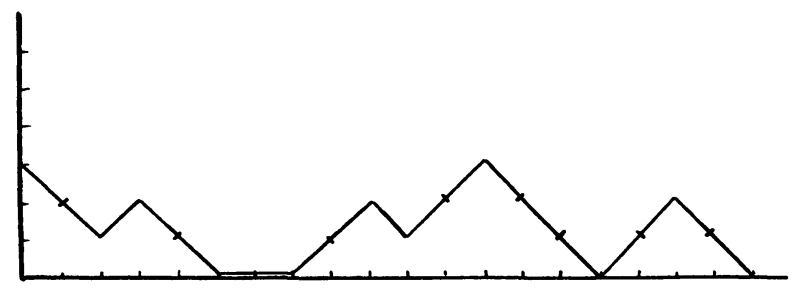

FIGURE 7

LEMMA 3.1. The function

$$
\frac{q^{m_{1}^{2}+\cdots+m_{r}^{2}}}{(q)_{m_{1}-m_{2}} \cdots(q)_{m_{r-1}-m_{r}}(q)_{m_{r}}\left(q ; q^{2}\right)_{m_{r}}}
$$


is the generating function for lattice paths starting at $(0,0)$ with no valleys above height $2 r-2=k-3$ and such that for $1 \leq i \leq r$ there are exactly $m_{i}$ relative peaks for which the difference of the coordinates is at least $i$ and the second coordinate is at most $2 r-2 i=k-2 i-1$.

Proof. We observe that this lemma implies that $m_{1}$ counts the number of peaks and that $m_{1} \geq m_{2} \geq \cdots \geq m_{r}$. When $r=1$, it agrees with the interpretation given for equation (2.1). The proof will proceed by establishing the inductive step on $r$.

Let $r^{\prime}=r-1, k^{\prime}=k-2$, and assume that

$$
\frac{q^{m_{2}^{2}+\cdots+m_{r}^{2}}}{(q)_{m_{2}-m_{3}} \cdots(q)_{m_{r-1}-m_{r}}(q)_{m_{r}}\left(q ; q^{2}\right)_{m_{r}}}
$$

is the generating function for lattice paths starting at $(0,0)$, with no valleys above height $k^{\prime}-3$ and such that for $2 \leq i \leq r$ there are exactly $m_{i}$ relative peaks for which the difference of the coordinates is at least $i-1$ and the second coordinate is at most $k^{\prime}-2(i-1)-1=k-2 i-1$.

We introduce the factor

$$
\frac{q^{m_{1}^{2}}}{(q)_{m_{1}-m_{2}}}
$$

The effect of this factor on the lattice path is first to increase the height of each peak by one so that, for example, the path

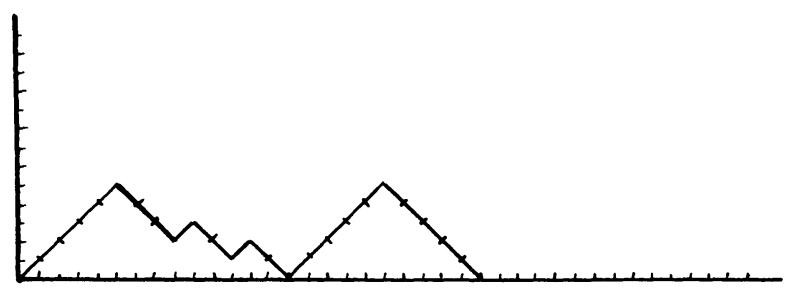

FIGURE 8

becomes

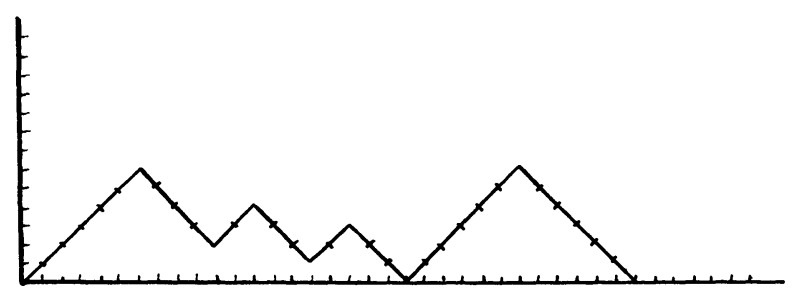

FIGURE 9 
This "volcanic action" increases the weight of the path by $1+3+\cdots+$ $\left(2 m_{2}-1\right)=m_{2}^{2}$. It also has the effect of increasing the first coordinate of all relative peaks by one so that the parameter $m_{i}$ now counts the number of relative peaks for which the difference of the coordinates is at least $i$ and the second coordinate is at most $k-2 i-1$.

We next insert the minimal path starting at $(0,0)$ with $m_{1}-m_{2}$ peaks of height one in front our our path. This increases the weight of the path by an additional $\left(m_{1}-m_{2}\right)^{2}+2 *\left(m-1-m_{2}\right) * m_{2}$, so that the total amount of increase to the weight of the path is

$$
m_{2}^{2}+\left(m_{1}-m_{2}\right)^{2}+2 *\left(m_{1}-m_{2}\right) * m_{2}=m_{1}^{2} .
$$

Our example becomes $\left(m_{1}-m_{2}=3\right)$ :

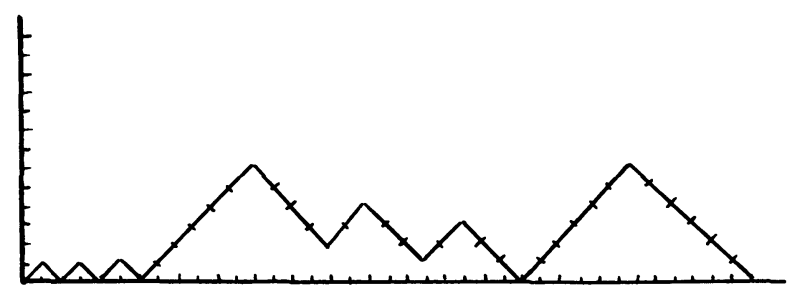

FIGURE 10

We note that $m_{1}$ now counts the total number of peaks and that none of the new peaks are counted by $m_{2}$.

The factor $(q)_{m_{1}-m_{2}}^{-1}$ gives us $m_{1}-m_{2}$ non-negative integers, say $a_{1} \geq a_{2} \geq \cdots \geq a_{m_{1}-m_{2}} \geq 0$. Beginning with the right-most of the new peaks, we move it to the right $a_{1}$ times according to the following rules. After each move the peak will still not be counted by $m_{2}$ which means that when viewed as a relative peak either the difference in the coordinates is one or the second coordinate is $k-3$ or $k-4$.

(1) If the peak is followed by a southeast step followed by a horizontal step, we change the preceding northeast step to a horizontal step, the southeast step to northeast and the horizontal step to southeast.

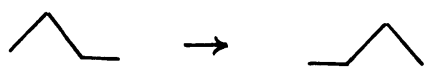

FIGURE 11

(2) If the peak is followed by two southeast steps and it is preceded by a northeast step preceded by a southeast step, then we change the 
preceding northeast step to southeast and the following southeast step to northeast.

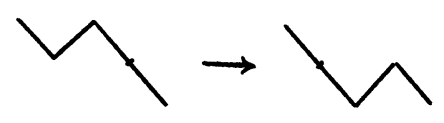

Figure 12

(3) If the peak is followed by a southeast step followed by two northeast steps and its height is not more than $k-3$, we change the southeast step to northeast and the first of the following northeast steps to southeast.

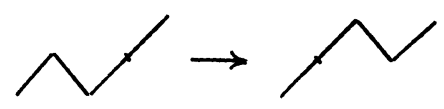

FIGURE 13

(4) If the peak is followed by a southeast step followed by two northeast steps and its height is at least $k-2$, then we change the first northeast step following the southeast step to southeast and change the first southeast step after the next peak to a northeast step. Note that since all valleys have height at most $k-3$, the next peak is followed by at least two southeast steps. Also note that while the peak being moved is now a relative peak whose difference of coordinates is at least two, the second coordinate is $k-4$ so that it is not counted by $m_{2}$.

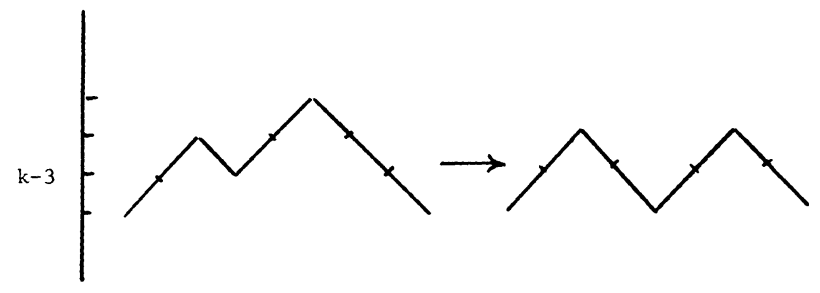

FIGURE 14

(5) If the peak is preceded by at least two northeast steps and followed by at least two southeast steps, then there is a valley on at least one side of the peak which has height $k-3$ or $k-4$. We consider five subcases:

(a) If the valley to the right has height $k-3$ and the next peak is higher than the peak being moved, then we change the first northeast 
step between the peaks to southeast, and the first southeast step after the second peak to northeast.

(b) If the valley to the right has height $k-4$ and the next peak is higher than the peak being moved, then we change the first southeast step after the first peak to northeast and the first northeast step between the peaks to southeast.

(c) If the valley to the right has height $k-3$ or $k-4$ and the next peak is the same height as the peak being moved, we shift our attention to the peak on the right and instead move it.

(d) If the valley to the right has height less than $k-4$ and the valley to the left has height $k-3$, then we change the first northeast step between the peak to the left and the peak being moved to southeast and the first southeast step after the peak being moved to northeast.

(e) If the valley to the right has height less than $k-4$ and the valley to the left has height $k-4$, then we change the first southeast step after the peak to the left to northeast and the first northeast step between the peak to the left and peak being moved to southeast.

All of these possibilities are illustrated in the next example where we move the peak by four. For purposes of identification, we place a dot in the peak being moved.
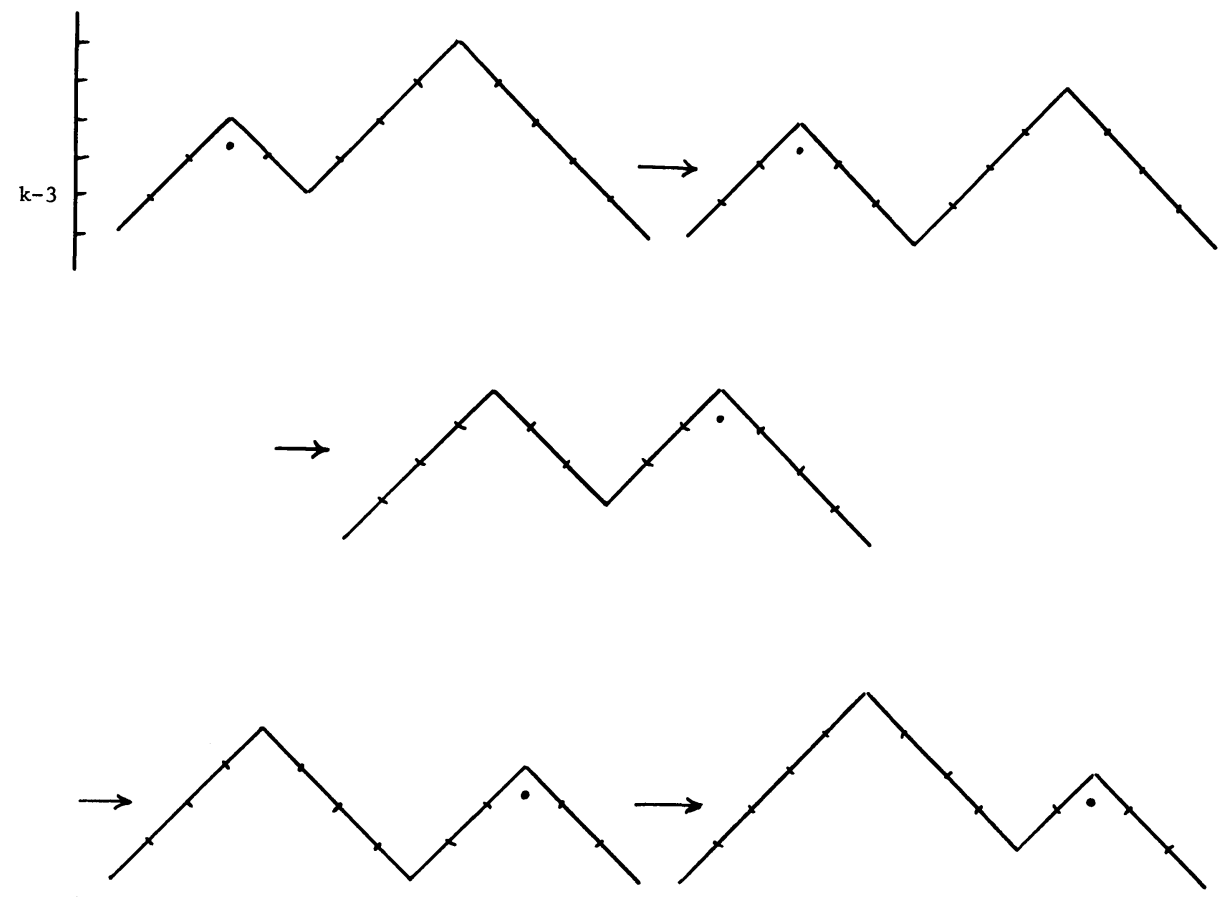

Figure 15 
(6) If the peak is followed by a southeast step followed by a northeast step followed by a southeast step, we shift our attention to the peak on the right and instead move it.

In our example, we assume that $k=7$ and that $a_{1}=10$. The dot marks the final position of the peak being moved.

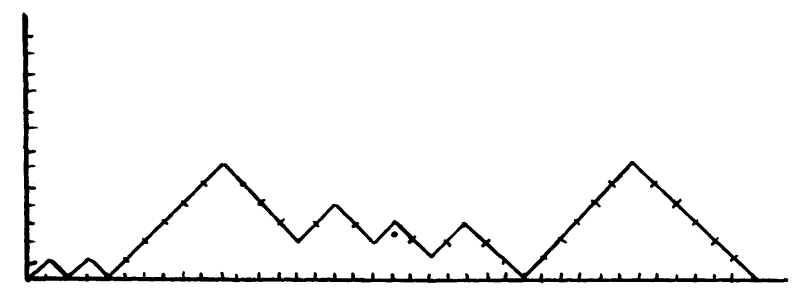

\section{Figure 16}

We proceed in similar manner for each $a_{i}$. After moving the $(i-1)$ st new peak, counted from the right, $a_{i-1}$ steps to the right, we move the next new peak $a_{i}$ steps to the right. Since the $a_{i}$ 's are weakly decreasing, no new peak ever moves over another new peak.

It should be clear that every path starting at $(0,0)$, with no valleys above height $k-3$ and with constants $m_{1}, m_{2}, \ldots, m_{r}$ as described above can be uniquely created in this way, thus concluding the proof of this lemma.

It follows from Lemma 3.1 that summing over all choices of $m-1 \geq$ $m_{2} \geq \cdots \geq m_{r} \geq 0$ yields the generating function for all lattice paths starting at $(0,0)$ which have no valleys above height $k-3$, thus proving Proposition 1.4 for equation (1.7).

To prove the proposition for equation (1.8) we use an analogous lemma.

LEMMA 3.2. The function

$$
\frac{q^{m_{1}^{2}+\cdots+m_{r}^{2}+m_{r}\left(m_{r}-1\right) / 2}}{(q)_{m_{1}-m_{2}} \cdots(q)_{m_{r-1}-m_{r}}(q)_{m_{r}}\left(q ; q^{2}\right)_{m_{r}}}
$$

is the generating function for lattice paths starting at $(0,0)$ with no valleys above height $2 r-3=k-3$ and such that for $1 \leq i \leq r$ there are exactly $m_{i}$ relative peaks for which the difference of the coordinates is at least $i$ and the second coordinate is at most $2 r-2 i-1=k-2 i-1$.

Proof. The proof proceeds essentially the same as the proof of Lemma 3.1. The only difference is that the factor of $q^{m_{r}\left(m_{r}-1\right) / 2}$ will 
introduce a horizontal step between each pair of original peaks. Note that in the inductive step, $k$ is at least four.

Before leaving this section, it is worth noting that for the generating function in Lemma 3.1, $m_{r}$ is the number of mountains of height at least $r$. For the generating function in Lemma 3.2, $m_{r}$ is the number of ranges of height at least $r$.

4. Proof of Proposition 1.4 for equations (1.5) and (1.6). To interpret the left hand side of equation (1.5) we first observe that by the argument at the end of $\S 2$ together with Lemma 3.1, we have that the function

$$
\frac{q^{m_{t}^{2}+\cdots+m_{r}^{2}-m_{t}}\left(1-q^{m_{t}}\right)}{(q)_{m_{t}-m_{t+1}} \cdots(q)_{m_{r-1}-m_{r}}(q)_{m_{r}}\left(q ; q^{2}\right)_{m_{r}}},
$$

where $t=k-i$, is the generating function for lattice paths starting at $(0,1)$ which have no valleys above height $2(r-(k-i)+1)+1-3=$ $2 i-k-1$ and $m_{j}, k-i \leq j \leq r$, counts the number of relative peaks for which the difference between the first and second coordinate is at least $j+i-k+1$ and whose second coordinate is at most $k-2 j-1$. This establishes the case $h=k-i$ of the following lemma which we can then prove by induction.

LEMMA 4.1. For $1 \leq h \leq k-i \leq(k-1) / 2=r, t=k-i$, the function

$$
\frac{q^{m_{h}^{2}+\cdots+m_{r}^{2}-m_{h}-\cdots-m_{t}}\left(1-q^{m_{t}}\right)}{(q)_{m_{h}-m_{h+1}} \cdots(q)_{m_{r-1}-m_{r}}(q)_{m_{r}}\left(q ; q^{2}\right)_{m_{r}}}
$$

is the generating function for lattice paths starting at $(0, k-i-h+1)$, which have no valleys above height $k-2 h-1$ and for which $m_{j}$ counts the number of relative peaks for which the difference between the first and second coordinate is at least $j-h+1$ and the second coordinate is at most $k-2 j-1$.

Proof. We assume the lemma is true for $h+1$ and prove that it also holds for $h$. The factor

$$
\frac{q^{m_{h}^{2}-m_{h}}}{(q)_{m_{h}-m_{h+1}}}
$$

is encoded as follows. Each pre-existing peak is increased in height by one, increasing the weight of the path by $m_{h+1}^{2}$. We then insert at the front of the path of $m_{h}-m_{h+1}$ peaks connecting the vertices:

$$
\begin{aligned}
&(0, k-i-h+1),(1, k-i-h), \quad(2, k-i-h+1), \\
&(3, k-i-h), \ldots,\left(2\left(m_{h}-m_{h+1}\right)-1, k-i-h\right) .
\end{aligned}
$$


This further increases the weight of the path by

$$
\begin{gathered}
\left(m_{h}-m_{h+1}\right) *\left(m_{h}-m_{h+1}-1\right)+\left(2^{*}\left(m_{h}-m_{h+1}\right)-1\right) * m_{h+1} \\
=m_{h}^{2}-m_{h+1}^{2}-m_{h},
\end{gathered}
$$

accounting for the factor of $q^{m_{h}^{2}-m_{h}}$.

The factor $(q)_{m_{h}-m_{h+1}}^{-1}$ is used to move the new peaks to the right in exactly the same manner as in $\S 3$.

A similar lemma, established in exactly the same way, proves Proposition 1.4 for equation (1.6).

LEMMA 4.2. For $1 \leq h \leq k-i \leq k / 2=r, t=k-i$, the function

$$
\frac{q^{m_{h}^{2}+\cdots+m_{r}^{2}-m_{h}-\cdots-m_{t}+m_{t}\left(m_{t}-1\right) / 2}\left(1-q^{m_{t}}\right)}{(q)_{m_{h}-m_{h+1}} \cdots(q)_{m_{r-1}-m_{r}}(q)_{m_{r}}\left(q ; q^{2}\right)_{m_{r}}}
$$

is the generating function for lattice paths starting at $(k-i-h+1,0)$, which have no valleys above height $k-2 h-1$ and for which $m_{j}$ counts the number of relative peaks for which the difference between the first and second coordinate is at least $j-h+1$ and whose second coordinate is at most $k-2 j-1$.

5. Proof of Proposition 1.4 for equations (1.3) and (1.4). The next two lemmas will conclude the proof of Proposition 1.4.

LEMMA 5.1. For $1 \leq i \leq r+1=(k+1) / 2$, the function

$$
\frac{q^{m_{1}^{2}+\cdots+m_{r}^{2}+m_{1}+\cdots+m_{1-1}+2 m_{1}+\cdots+2 m_{r}}}{(q)_{m_{1}-m_{2}} \cdots(q)_{m_{r-1}-m_{r}}(q)_{m_{r}}\left(q ; q^{2}\right)_{m_{r}+1}}
$$

is the generating function for lattice paths starting at height $k-i$, with no valleys above height $k-3$ and such that for $1 \leq j \leq r$ there are exactly $m_{j}+1$ relative peaks whose difference between first and second coordinates is at least $j$ and whose second coordinate is at most $k-$ $2 j-1$. The number of mountains of height at least $r$ is $m_{r}+1$.

Proof. When $r=1$ this is the result proved in $\S 2$. We proceed by decreasing induction on $r$. Assume the lemma is true for $r-1$. Let $k^{\prime}=k-2, i^{\prime}=i-1$ if $i$ is at least two, $i^{\prime}=1$ if $i=1$. Then $1 \leq i^{\prime} \leq\left(k^{\prime}+1\right) / 2$. Our induction hypothesis is that

$$
\frac{q^{m_{2}^{2}+\cdots+m_{r}^{2}+m_{2}+\cdots+m_{i^{\prime}}+2 m_{1^{\prime}+1}+\cdots+2 m_{r}}}{(q)_{m_{2}-m_{3}} \cdots(q)_{m_{r-1}-m_{r}}(q)_{m_{r}}\left(q ; q^{2}\right)_{m_{r}+1}}
$$


is the generating function for lattice paths starting at $\left(0, k^{\prime}-i^{\prime}\right)$ with no valleys above height $k^{\prime}-3=k-5$ and such that for $2 \leq j \leq r$ there are exactly $m_{j}+1$ relative peaks for which the difference in coordinates is at least $j-1$ and whose second coordinate is at most $k^{\prime}-2(j-1)-1=k-2 j-1$.

If $i$ is larger than one, then the new factor is

$$
\frac{q^{m_{1}^{2}+m_{1}}}{(q)_{m_{1}-m_{2}}}
$$

As before, increasing the height of each of the $m_{2}+1$ existing peaks by one and then inserting the lattice path of $m_{1}-m_{2}$ peaks starting at $(0, k-i)$ and ending at $\left(2\left(m_{1}-m_{2}\right)-1, k-i-1\right)$ accounts for the increase in weight of $m_{1}^{2}+m_{1}$. The factor $(q)_{m_{1}-m_{2}}^{-1}$ is used to move the new peaks to the right.

If $i$ equals one, then the pre-existing path started at $(0, k-3)$. We increase each of the pre-existing peaks by one and then insert the lattice path of $m_{1}-m_{2}$ peaks starting at $(0, k-1)$, passing through $(2, k-3)$ and ending at $\left(2\left(m_{1}-m_{2}\right), k-3\right)$. This increases the weight of the path by $m_{1}^{2}+2 m_{1}$. The factor $(q)_{m_{1}-m_{2}}^{-1}$ is used to move the new peaks to the right.

LEMMA 5.2. For $1 \leq i \leq r+1=(k+2) / 2$, the function

$$
\frac{q^{m_{1}^{2}+\cdots+m_{r}^{2}+m_{1}+\cdots+m_{l-1}+2 m_{i}+\cdots+2 m_{r}+m_{r}\left(m_{r}-1\right) / 2}}{(q)_{m_{1}-m_{2}} \cdots(q)_{m_{r-1} m_{r}}(q)_{m_{r}}\left(q ; q^{2}\right)_{m_{r}}}
$$

is the generating function for lattice paths starting at height $k-i$, with no valleys above height $k-3$ and such that for $1 \leq j<r$ there are exactly $m_{j}+1$ relative peaks whose difference between first and second coordinates is at least $j$ and whose second coordinate is at most $k-$ $2 j-1$. The parameter $m_{r}$ counts the number of ranges of height at least $r$ which follow the first plain.

Proof. Again, this is proved exactly as Lemma 5.1 except that we start with at least one horizontal step in front of each of the initial $m_{r}$ mountains.

6. Partitions with " $n+t$ copies of $n$ ". If we specify the constants $k$ and $i, 1 \leq i \leq k$, and are then given a lattice path which begins at height $k-i$ and has no valleys above height $k-3$, we can encode this path as the sequence of the weights of the peaks with each weight 
subscripted by the height of the respective peak. Thus the path

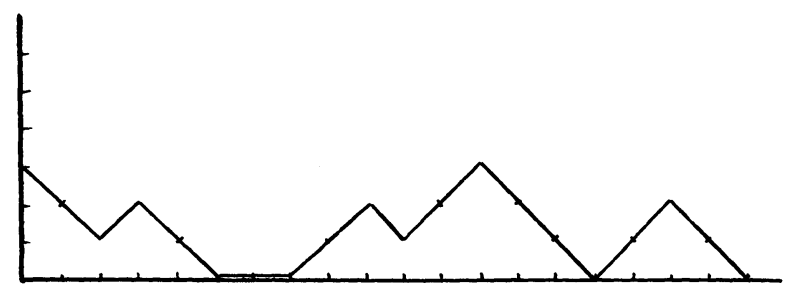

FIGURE 17

is encoded as $\left(0_{3}, 3_{2}, 9_{2}, 12_{3}, 17_{2}\right)$. Given this encoding and the parameters $k$ and $i$, the path is uniquely reconstructible.

It should be obvious that not every finite increasing sequence of subscripted non-negative integers corresponds to one of our lattice paths. First of all, the subscripts are always at least one and the subscript for an integer $n$ cannot be larger than $n+k-i$. If we denote the constant $k-i$ by $t$, this means that we have $n+t$ possible subscripts for any given non-negative integer $n$. Agarwal and Andrews [A\&A] have described the sequences which correspond to our lattice paths as restricted partitions with $n+t$ copies of $n$. This is somewhat misleading as no weight can be repeated. The next proposition provides the link between the partitions with $n+t$ copies of $n$ as defined by Agarwal and Andrews and our lattice paths.

Proposition 6.1. Given $k$ and $i, 1 \leq i \leq k$, let $t=k-i$. A finite increasing sequence of subscripted non-negative integers corresponds to a lattice path starting at $(0, t)$ and with no valleys above height $k-3$ if and only if the sequence satisfies the following properties:

(1) The subscript on each integer $n$ lies in the range $[1, n+t]$.

(2) If $t>0$, then the subscript on the first term of the sequence is exactly $t$ more than the value of that entry.

The remaining conditions apply to every pair $a_{x}, b_{y}$ of consecutive terms in the sequence.

(3) $b-a \geq|x-y|+2$.

(4) If $b-a \leq x+y$, then $a+x$ and $b+y$ have the same parity.

(5) If $k \geq 3$, then $b-a \geq x+y-2 k+6$.

(6) If $k=2$, then $b-a \geq x+y+1$.

Proof. The first property was explained above. For property 2, if the path starts at $(0, t), t>0$, then the first southeast step defines the first peak and the height of this peak will be $t$ more than its weight. 
Property 3 says that the weights of two successive peaks differ by at least two more than the absolute difference in heights. This follows from the fact that between these peaks one must descend to a height at or below the minimum of $x-1$ and $y-1$.

For property 4, if the weights of two successive peaks differ by at most the sum of the heights, then these two peaks must lie in the same range. All vertices on a given range have the same parity for the sum of height and weight.

Since no valley lies above height $k-3$, if $k \geq 3$ then to get from one peak to the next one must descend at least to height $k-3$ (a descent of at least $x-(k-3)$ ) and then ascend from at most height $k-3$ (an ascent of at least $y-(k-3))$. Thus the difference in weights is at least

$$
x-(k-3)+y-(k-3)=x+y-2 k+6 .
$$

If $k=2$, then to get from one peak to the next one must descend to height 0 , make at least one horizontal step and then ascend to the next peak so that the difference in weights of the peaks is at least $x+1+y$.

We leave it to the readers to convince themselves that properties 14 describe all sequences corresponding to lattice paths which start at $(0, t)$ and have no other restrictions. Properties 5 and 6 are precisely what guarantees that all valleys descend to height $k-3$ or below.

Acknowledgment. We wish to thank Basil Gordon for his helpful comments and suggestions on the presentation of this paper.

\section{REFERENCES}

[A\&A] A. K. Agarwal and G. E. Andrews, Rogers-Ramanujan identities for partitions with " $N+t$ copies of $N$ ", to appear.

[AA\&B] A. K. Agarwal, G. E. Andrews and D. M. Bressoud, The Bailey lattice, J. Indian Math. Soc., to appear.

[And 1] G. E. Andrews, An analytic generalization of the Rogers-Ramanujan identities for odd moduli, Proc. Nat. Acad. Sci. USA, 71 (1974), 4082-4085.

[And 2] ㄴ Sieves in the theory of partitions, Amer. J. Math., 94 (1972), 12141230.

[A\&B] G. E. Andrews and D. M. Bressoud, On the Burge correspondence between partitions and binary words, Rocky Mtn. J. Math., 15 (1985), 225-233.

[Bre 1] D. M. Bressoud, An analytic generalization of the Rogers-Ramanujan identities with interpretation, Quart. J. Math. Oxford (2), 31 (1980), 385-399.

[Bre 2] _ Analytic and combinatorial generalization of the Rogers-Ramanujan identities, Memoirs Amer. Math. Soc., 24 (1980), no. 227.

[Bur 1] W. H. Burge, $A$ correspondence between partitions related to generalizations of the Rogers-Ramanujan identities, Discrete Math., 34 (1981), 915. 
[Bur 2] _ A three-way correspondence between partitions, Europ. J. Combinatorics, 3 (1982), 195-213.

[Gor] B. Gordon, A combinatorial generalization of the Rogers-Ramunujan identities, Amer. J. Math., 83 (1961), 393-399.

Received February 9, 1987 and in revised form March 4, 1988. The second author was partially supported by NSF Grant No. DMS-8521580.

Pennsylvania State University

UNIVERSITy PARK, PA 16802 


\section{PACIFIC JOURNAL OF MATHEMATICS \\ EDITORS}

\author{
V. S. VARADARAJAN \\ (Managing Editor) \\ University of California \\ Los Angeles, CA 90024 \\ HERBERT ClEMENS \\ University of Utah \\ Salt Lake City, UT 84112 \\ THOMAS ENRIGHT \\ University of California, San Diego \\ La Jolla, CA 92093
}

R. FINN

Stanford University

Stanford, CA 94305

HERMANN FLASCHKA

University of Arizona

Tucson, AZ 85721

VAUGhan F. R. JONES

University of California

Berkeley, CA 94720

STEVEN KERCKHOFF

Stanford University

Stanford, CA 94305

\section{ROBION KIRBY}

University of California

Berkeley, CA 94720

C. C. MOORE

University of California

Berkeley, CA 94720

HAROLD STARK

University of California, San Diego

La Jolla, CA 92093

\section{ASSOCIATE EDITORS}
R. ARENS
E. F. BECKENBACH
B. H. NEUMANN
F. WOLF
K. YOSHIDA

(1906-1982)

\section{SUPPORTING INSTITUTIONS}

UNIVERSITY OF ARIZONA

UNIVERSITY OF BRITISH COLUMBIA

CALIFORNIA INSTITUTE OF TECHNOLOGY

UNIVERSITY OF CALIFORNIA

MONTANA STATE UNIVERSITY

UNIVERSITY OF NEVADA, RENO

NEW MEXICO STATE UNIVERSITY

OREGON STATE UNIVERSITY

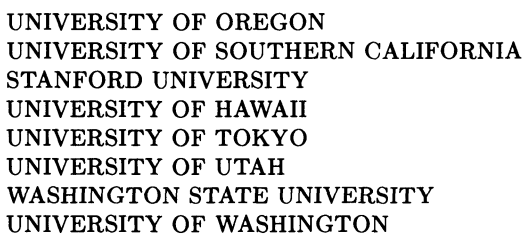

The Supporting Institutions listed above contribute to the cost of publication of this Journal, but they are not owners or publishers and have no responsibility for its content or policies.

\footnotetext{
Mathematical papers intended for publication in the Pacific Journal of Mathematics should be in typed form or offset-reproduced (not dittoed), double spaced with large margins. Please do not use built up fractions in the text of the manuscript. However, you may use them in the displayed equations. Underline Greek letters in red, German in green, and script in blue. The first paragraph must be capable of being used separately as a synopsis of the entire paper. In particular it should contain no bibliographic references. Please propose a heading for the odd numbered pages of less than 35 characters. Manuscripts, in triplicate, may be sent to any one of the editors. Please classify according to the scheme of Math. Reviews, Index to Vol. 39. Supply name and address of author to whom proofs should be sent. All other communications should be addressed to the managing editor, or Elaine Barth, University of California, Los Angeles, California 90024.

There are page-charges associated with articles appearing in the Pacific Journal of Mathematics. These charges are expected to be paid by the author's University, Government Agency or Company. If the author or authors do not have access to such Institutional support these charges are waived. Single authors will receive 50 free reprints; joint authors will receive a total of 100 free reprints. Additional copies may be obtained at cost in multiples of 50 .
}

The Pacific Journal of Mathematics is issued monthly as of January 1966. Regular subscription rate: $\$ 190.00$ a year (5 Vols., 10 issues). Special rate: $\$ 95.00$ a year to individual members of supporting institutions.

Subscriptions, orders for numbers issued in the last three calendar years, and changes of address should be sent to Pacific Journal of Mathematics, P.O. Box 969, Carmel Valley, CA 93924, U.S.A. Old back numbers obtainable from Kraus Periodicals Co., Route 100, Millwood, NY 10546.

The Pacific Journal of Mathematics at P.O. Box 969, Carmel Valley, CA 93924 (ISSN 0030-8730) publishes 5 volumes per year. Application to mail at Second-class postage rates is pending at Carmel Valley, California, and additional mailing offices. Postmaster: send address changes to Pacific Journal of Mathematics, P.O. Box 969, Carmel Valley, CA 93924.

PUBLISHED BY PACIFIC JOURNAL OF MATHEMATICS, A NON-PROFIT CORPORATION Copyright (C) 1989 by Pacific Journal of Mathematics 


\section{Pacific Journal of Mathematics}

\section{Vol. 136, No. 2 December, 1989}

A. K. Agarwal and David Bressoud, Lattice paths and multiple basic hypergeometric series ................................. 209

Adalberto Panobianco Bergamasco and Hermano de Souza Ribeiro, Uniqueness in a doubly characteristic Cauchy problem ........... 229

Thomas Curtis Craven and George Leslie Csordas, Jensen polynomials

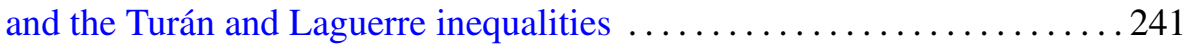

Gary R. Jensen and Marco Rigoli, Harmonic Gauss maps ............ 261

L. G. Kovács and Cheryl Elisabeth Praeger, Finite permutation groups with large abelian quotients $\ldots \ldots \ldots \ldots \ldots \ldots \ldots \ldots \ldots \ldots \ldots \ldots . \ldots \ldots$

Ken-ichi Maruyama, Localization of a certain subgroup of self-homotopy

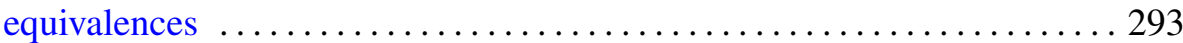

Tomasz Mazur, Canonical isometry on weighted Bergman spaces ......... 303

Bernt Karsten Oksendal, A stochastic Fatou theorem for quasiregular

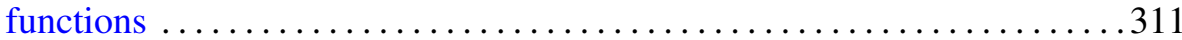

Ian Fraser Putnam, The $C^{*}$-algebras associated with minimal homeomorphisms of the Cantor set ........................ 329

Tom Joseph Taylor, Some aspects of differential geometry associated with

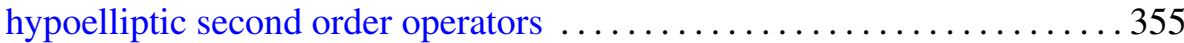

Tom Joseph Taylor, Off diagonal asymptotics of hypoelliptic diffusion equations and singular Riemannian geometry 